\title{
Constraint Answer Set Programming without Grounding*
}

\author{
JOAQUIN ARIAS, MANUEL CARRO \\ IMDEA Software Institute and Universidad Politécnica de Madrid \\ (e-mail: joaquin.arias@\{imdea.org, alumnos.upm.es $\}$, manuel.carro@\{imdea.org, upm.es)
}

\author{
ELMER SALAZAR, KYLE MARPLE, and GOPAL GUPTA \\ University of Texas at Dallas \\ (e-mail: ees101020,kmarple1, gupta\}@utdallas.edu)
}

submitted 03 May 2018; accepted 11 May 2018

\begin{abstract}
Extending ASP with constraints (CASP) enhances its expressiveness and performance. This extension is not straightforward as the grounding phase, present in most ASP systems, removes variables and the links among them, and also causes a combinatorial explosion in the size of the program. Several methods to overcome this issue have been devised: restricting the constraint domains (e.g., discrete instead of dense), or the type (or number) of models that can be returned. In this paper we propose to incorporate constraints into $\mathrm{s}(\mathrm{ASP})$, a goal-directed, top-down execution model which implements ASP while retaining logical variables both during execution and in the answer sets. The resulting model, s(CASP), can constrain variables that, as in CLP, are kept during the execution and in the answer sets. s(CASP) inherits and generalizes the execution model of S(ASP) and is parametric w.r.t. the constraint solver. We describe this novel execution model and show through several examples the enhanced expressiveness of s(CASP) w.r.t. ASP, CLP, and other CASP systems. We also report improved performance w.r.t. other very mature, highly optimized ASP systems in some benchmarks.
\end{abstract}

\section{Introduction}

Answer Set Programming (ASP) has emerged as a successful paradigm for developing intelligent applications. It uses the stable model semantics (Gelfond and Lifschitz 1988) for programs with negation. ASP has attracted much attention due to its expressiveness, ability to incorporate non-monotonicity, represent knowledge, and model combinatorial problems. On the other hand, constraints have been used both to enhance expressiveness and to increase performance in logic programming. Therefore, it is natural to incorporate constraints in ASP systems. This is however not straightforward as ASP systems usually carry out an initial grounding phase where variables (and, therefore, the constraints linking them) disappear. Several approaches have been devised to work around this issue. However, since constraints need to be grounded as well, these approaches limit the range of admissible constraint domains (e.g., discrete instead of dense), the places where constraints can appear, and the type (or number) of models that can be returned. The integration of constraints with ASP is not as seamless as in standard constraint logic programming (CLP).

* Work partially supported by EIT Digital (https://eitdigital.eu), MINECO project TIN2015-67522-C3-1-R (TRACES), Comunidad de Madrid project S2013/ICE-2731 N-Greens Software, NSF IIS 1718945, and NSF IIS 1423419. 
In this work we propose to restore this integration by incorporating constraints into the s(ASP) (Marple et al. 2017b) execution model. s(ASP) is a goal-directed, top-down, SLD resolution-like procedure which evaluates programs under the ASP semantics without a grounding phase either before or during execution. s(ASP) supports predicates and thus retains logical variables both during execution and in the answer sets. We have extended s(ASP)'s execution model to make its integration with generic constraint solvers possible. The resulting execution model and system, called s(CASP), makes it possible to express constraints on variables and extends s(ASP)'s in the same way that CLP extends Prolog's execution model. Thus, s(CASP) inherits and generalizes the execution model of s(ASP) while remaining parametric w.r.t. the constraint solver. Due to its basis in s(ASP), s(CASP) avoids grounding the program and the concomitant combinatorial explosion. s(CASP) can also handle answer set programs that manipulate arbitrary data structures as well as reals, rationals, etc. We show, through several examples, its enhanced expressiveness w.r.t. ASP, CLP, and other ASP systems featuring constraints. We briefly discuss s(CASP)'s efficiency: on some benchmarks it can outperform mature, highly optimized ASP systems.

Several approaches have been proposed to mitigate the impact of the grounding phase in ASP systems. In the case of large data sets, magic set techniques have been used to improve grounding for specific queries (Alviano et al. 2012). For programs which use uninterpreted function symbols, techniques such as external sources (Calimeri et al. 2007) have been proposed.

Despite these approaches, grounding is still an issue when constraints are used in ASP. Variable domains induced by constraints can be unbound and, therefore, infinite (e.g., $X \#>0$ with $X \in \mathbb{N}$ or $X \in \mathbb{Q}$ ). Even if they are bound, they can contain an infinite number of elements (e.g. $X \#>0$

$\wedge \mathrm{X} \#<1$ in $\mathbb{Q}$ or $\mathbb{R}$ ). These problems have been attacked using different techniques:

- Translation-based methods (Balduccini and Lierler 2017), which convert both ASP and constraints into a theory that is executed in an SMT solver-like manner. Once the input program is translated, they benefit from the features and performance of the target ASP and CLP solvers. However, the translation may result in a large propositional representation or weak propagation strength.

- Extensions of ASP systems with constraint propagators (Banbara et al. 2017; Janhunen et al. 2017) that generate and propagate new constraints during the search and thus continuously check for consistency using external solvers using e.g. conflict-driven clause learning. However, they are restricted to finite domain solvers (hence, dense domains cannot be appropriately captured) and incrementally generate ground models, lifting the upper bounds for some parameters. This, besides being a performance bottleneck, falls short of capturing the true nature of variables in constraint programming.

Due to the requirement to ground the program, causing a loss of communication from elimination of variables, the execution methods for CASP systems are complex. Explicit hooks sometimes are needed in the language, e.g., the required builtin of EZCSP (Balduccini and Lierler 2017), so that the ASP solver and the constraint solver can communicate. Additionally, considerable research has been conducted on devising top-down execution models for ASP (Dal Palù et al. 2009; Baselice et al. 2009; Baselice and Bonatti 2010) that could be extended with constraints. 
We have validated the s(CASP) approach with an implementation in Ciao Prolog which integrates Holzbaur's CLP(Q) (Holzbaur 1995), a linear constraint solver over the rationals. ${ }^{1}$ The $\mathrm{s}$ (CASP) system has been used to solve a series of problems that would cause infinite recursion in other top-down systems, but which in s(CASP) finitely finish, as well as others that require constraints over dense and/or unbound domains. Thus, s(CASP) is able to solve problems that cannot be straightforwardly solved in other systems.

\section{Background: ASP and s(ASP)}

ASP (Gelfond and Lifschitz 1988; Brewka et al. 2011) is a logic programming and modelling language. An ASP program $\Pi$ is a finite set of rules. Each rule $r \in \Pi$ is of the form:

$$
a \leftarrow b_{1} \wedge \ldots \wedge b_{m} \wedge \text { not } b_{m+1} \wedge \ldots \wedge \text { not } b_{n} .
$$

where $a$ and $b_{1}, \ldots, b_{n}$ are atoms and not corresponds to default negation. An atom is an expression of form $p\left(t_{1}, \ldots, t_{n}\right)$ where $p$ is a predicate symbol of arity $n$ and $t_{i}$, are terms. An atom is ground if no variables occur in it. The set of all constants appearing in $\Pi$ is denoted by $C_{\Pi}$. The head of rule $r$ is $h(r)=\{a\}^{2}$ and the body consists of positive atoms $b^{+}(r)=\left\{b_{1}, \ldots, b_{m}\right\}$ and negative atoms $b^{-}(r)=\left\{b_{m+1}, \ldots, b_{n}\right\}$. Intuitively, rule $r$ is a justification to derive that $a$ is true if all atoms in $b^{+}(r)$ have a derivation and no atom in $b^{-}(r)$ has a derivation. An interpretation $I$ is a subset of the program's Herbrand base and it is said to satisfy a rule $r$ if $h(r)$ can be derived from $I$. A model of a set of rules is an interpretation that satisfies each rule in the set. An answer set of a program $\Pi$ is a minimal model (in the set-theoretic sense) of the program

$$
\Pi^{I}=\left\{h(r) \leftarrow b^{+}(r) \mid r \in \Pi, b^{-}(r) \cap I=\emptyset\right\}
$$

which is called the Gelfond-Lifschitz reduct of $\Pi$ with respect to I (Gelfond and Lifschitz 1991). The set of all answer sets of $\Pi$ is denoted by $A S(\Pi)$. ASP solvers which compute the answer sets of non-ground programs use the above semantics by first applying, to each rule $r \in \Pi$, all possible substitution from the variables in $r$ to elements of $C_{\Pi}$ (this procedure is called grounding). To make this grounding possible, the rules of the program should be safe, i.e., all variables that appear in a rule have to appear in some positive literal in the body. The rule is termed unsafe otherwise.

A difference between ASP and Prolog-style (i.e., SLD resolution-based) languages is the treatment of negated literals. Negated literals in a body are treated in ASP using their logical semantics based on computing stable models. The negation as failure rule of Prolog (i.e., SLDNF resolution (Clark 1978)) makes a negated call succeed (respectively, fail) iff the non-negated call fails (respectively, succeeds). To ensure soundness, SLDNF has to be restricted to ground calls, as a successful negated goal cannot return bindings. However, SLDNF increases the cases of non-termination w.r.t. SLD.

S(ASP) (Marple et al. 2017a, 2017b) is a top-down, goal-driven interpreter of ASP programs written in Prolog (http://sasp-system.sourceforge.net). The top-down evaluation makes the grounding phase unnecessary. The execution of an s(ASP) program starts with a query, and each answer is the resulting $m g u$ of a successful derivation, its justification, and a (partial) stable

1 Note that while we used $\operatorname{CLP}(\mathbb{Q})$ in this paper, $\operatorname{CLP}(\mathbb{R})$ could also have been used.

2 Disjunctive ASP programs (i.e., programs with disjunctions in the heads of rules) can be transformed into nondisjunctive ASP programs by using default negation (Ji et al. 2016). 
model. This partial stable model is a subset of the ASP stable model (Gelfond and Lifschitz 1988) including only the literals necessary to support the query with its output bindings. ${ }^{3}$

Example 1 (Assuming an extended Herbrand Base)

Given the program below:

1 married (john).

2 :- not married $(\mathrm{X})$.

most ASP systems are not able to compute its stable model (not even an empty one), because the global constraint is unsafe. On the other hand, s(ASP) is able to compute queries to programs with unsafe rules by assuming that the unsafe variables take values in an extended Herbrand Universe, and not just that of the terms which can be constructed from the symbols in the program. Therefore, using this alternative semantics :- not married(X). corresponds to $\neg \exists x$. $\neg$ married $(x) \equiv \forall x$.married $(x)$ and since the program only has evidence of one married individual ( $j \circ h n)$, there is no stable model (i.e., it cannot be derived that all possible individuals are married). However, if we add the (unsafe) fact married(X) (i.e., $\forall x$ xarried $(x)$ ) to the program, the resulting stable model will be $\{\operatorname{married}(\mathrm{X})\}$ - every element of the universe is married.

s(ASP) has two additional relevant differences w.r.t. Prolog: first, s(ASP) resolves negated atoms not $l_{i}$ against dual rules of the program (Section 2.1), instead of using negation as failure. This makes it possible for a non-ground negated call not $p(X)$ to return the results for which the positive call $\mathrm{p}(\mathrm{X})$ would fail. Second, and very important, the dual program is not interpreted under SLD semantics: a number of very relevant changes related to how loops are treated (see later) are introduced.

\subsection{Dual of a Logic Program}

The dual of a predicate $p / 1$ is another predicate that returns the $X$ such that $p(X)$ is not true. It is used to give a constructive answer to a goal not $p(X)$. The dual of a logic program is another logic program containing the dual of each predicate in the program (Alferes et al. 2004). To synthesize the dual of a logic program $P$ we first obtain Clark's completion (Clark 1978), which assumes that the rules of the program completely capture all possible ways for atomic formulas to be true, and then we apply De Morgan's laws:

1. For each literal $p / n$ that appears in the head of a rule, choose a tuple $\vec{x}$ of $n$ distinct, new variables $x_{1}, \ldots, x_{n}$.

2. For each $i$-th rule of a predicate $p / n$ of the form $p_{i}\left(\vec{t}_{i}\right) \leftarrow B_{i}$, with $i=1, \ldots, k$, make a list $\vec{y}_{i}$ of all variables that occur in the body $B_{i}$ but do not occur in the head $p_{i}\left(\vec{t}_{i}\right)$, add $\exists \vec{y}_{i}$ to the body and rename the variables that appear in the head $\vec{t}_{i}$ with the tuple $\vec{x}$, obtained in the previous step, resulting in a predicate representing $\forall \vec{x}\left(p_{i}(\vec{x}) \leftarrow \exists \vec{y}_{i} B_{i}\right)$. Note that $\vec{x}$ are local, fresh variables. This step captures the standard semantics of Horn clauses.

3. With all these rules and using Clark's completion, we form the sentences:

$$
\begin{aligned}
& \forall \vec{x}\left(p(\vec{x}) \longleftrightarrow p_{1}(\vec{x}) \vee \ldots \vee p_{k}(\vec{x})\right) \\
& \forall \vec{x}\left(p_{i}(\vec{x}) \longleftrightarrow \exists \vec{y}_{i}\left(b_{i .1} \wedge \ldots \wedge b_{i . m} \wedge \neg b_{i . m+1} \wedge \ldots \wedge \neg b_{i . n}\right)\right)
\end{aligned}
$$

3 Note that the subset property holds only when the Gelfond-Lifschitz transformation is applied assuming an extended Herbrand Base obtained by extending the set of constants in the program, $C_{\Pi}$, with an infinite number of new elements. 
4. Their semantically equivalent duals $\neg p / n, \neg p_{i} / n$ are:

$$
\begin{aligned}
& \forall \vec{x}\left(\neg p(\vec{x}) \longleftrightarrow \neg\left(p_{1}(\vec{x}) \vee \ldots \vee p_{k}(\vec{x})\right)\right) \\
& \forall \vec{x}\left(\neg p_{i}(\vec{x}) \longleftrightarrow \neg \exists \vec{y}_{i}\left(b_{i .1} \wedge \ldots \wedge b_{i . m} \wedge \neg b_{i . m+1} \wedge \ldots \wedge \neg b_{i . n}\right)\right)
\end{aligned}
$$

5. Applying De Morgan's laws we obtain:

$$
\begin{aligned}
& \forall \vec{x}\left(\neg p(\vec{x}) \longleftrightarrow \neg p_{1}(\vec{x}) \wedge \ldots \wedge \neg p_{k}(\vec{x})\right) \\
& \forall \vec{x}\left(\neg p_{i}(\vec{x}) \longleftrightarrow \forall \vec{y}_{i}\left(\neg b_{i .1} \vee \ldots \vee \neg b_{i . m} \vee b_{i . m+1} \vee \ldots \vee b_{i . n}\right)\right)
\end{aligned}
$$

which generates a definition for $\neg p(\vec{x})$ and a separate clause with head $\neg p_{i}(\vec{x})$ for each positive or negative literal $b_{i . j}$ in the disjunction. Additionally, a construction to implement the universal quantifier introduced in the body of the dual program is necessary (Section 2.3).

Definitions for the initially negated literals $\neg b_{i . m+1} \ldots \neg b_{i . n}$ and for each of the new negated literals $\neg b_{i .1} \ldots \neg b_{i . m}$ are similarly synthesized. At the end of the chain, unification has to be negated to obtain disequality, e.g., $x=y$ is transformed into $x \neq y$ (Section 2.2).

\section{Example 2}

Given the program below:

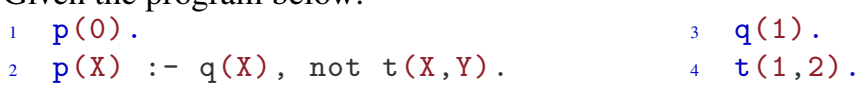

the resulting dual program is:

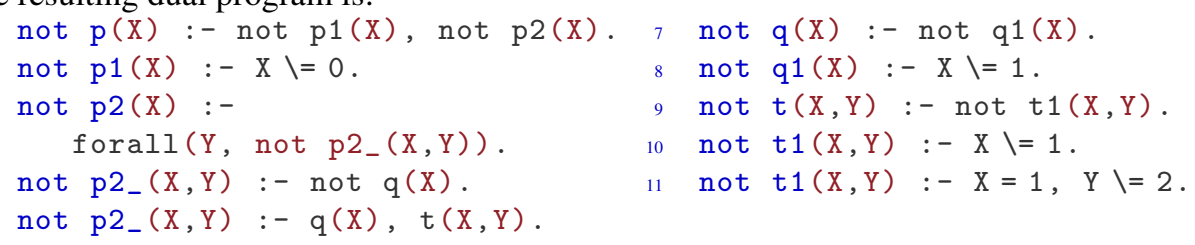

For efficiency, the generation of the dual diverges slightly from the previous scheme. The dual of a body $B \equiv l_{1} \wedge \ldots$ is the disjunction of its negated literals $\neg B \equiv \neg l_{1} \vee \ldots$, which generates independent clauses in the dual program. To avoid redundant answers, every clause for a negated literal $\neg l_{i}$ includes calls to any positive literal $l_{j}$ with $j<i$. E.g., clause 6 from the previous program, not $\mathrm{p} 2(\mathrm{X}, \mathrm{Y}):-\mathrm{q}(\mathrm{X}), \mathrm{t}(\mathrm{X}, \mathrm{Y})$, would only need to be not $\mathrm{p} 2(\mathrm{X}, \mathrm{Y}):-\mathrm{t}(\mathrm{X}, \mathrm{Y})$. However, the literal $\mathrm{q}(\mathrm{X})$ is included to avoid exploring solutions already provided by clause 5 , not $\mathrm{p} 2(\mathrm{X}, \mathrm{Y}):-$ not $\mathrm{q}(\mathrm{X})$. The same happens with clauses 10 and 11 .

\subsection{Constructive Disequality}

Unlike Prolog's negation as failure, disequality in s(ASP), denoted by " $\backslash=$ ", represents the constructive negation of the unification and is used to construct answers from negative literals. Intuitively, $X \backslash=a$ means that $X$ can be any term not unifiable with a. In the implementation reported in (Marple et al. 2017b) a variable can only be disequality-constrained against ground terms, and the disequality of two compound terms may require backtracking to check all the cases: $\mathrm{p}(1, \mathrm{Y}) \backslash=\mathrm{p}(\mathrm{X}, 2)$ first succeeds with $\mathrm{X} \backslash=1$ and then, upon backtracking, with $\mathrm{Y} \backslash=2$.

The former restriction reduces the range of valid programs, but this does not seem to be a problem in practice: since positive literals are called before negative literals in the dual program, the number of cases where this situation may occur is further reduced. Since this is orthogonal to the implementation framework, it can be improved upon separately. The second characteristic impacts performance, but can again be ameliorated with a more involved implementation of disequality which carries a disjunction of terms. 


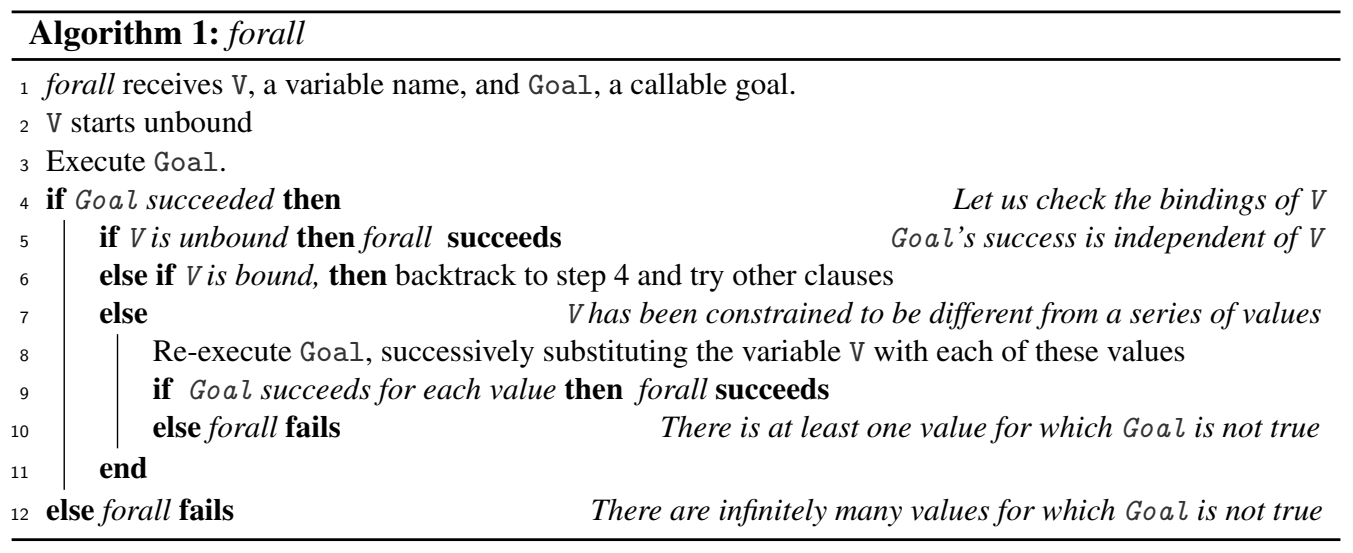

\subsection{Forall Algorithm}

In (Marple et al. 2017b) the universal quantifier is evaluated by forall (V, Goal) which checks if Goal is true for all the possible values of $\mathrm{V}$. When forall/2 succeeds, the evaluation continues with the quantified variable unbound. Multiple quantified variables are handled by nesting: $\forall v_{1}, v_{2}$.Goal is executed as forall (V1,forall (V2,Goal)). The underlying idea is to verify that for any solution with $\mathrm{V} \backslash=\mathrm{a}$ (for some a), Goal also succeeds with $\mathrm{V}=\mathrm{a}$ (Algorithm 1).

\section{Example 3}

Consider the following program with the dual rule for $\mathrm{p} / 0$ :

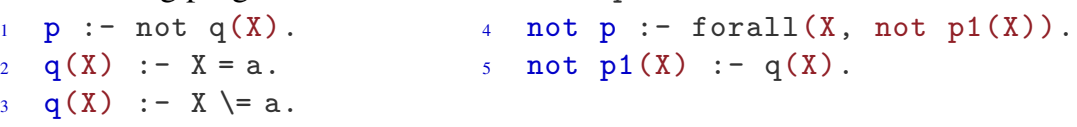

Under the query ?- not $p$, the interpreter will execute forall (X, not p1(X)) with $X$ unbound. First, not $\mathrm{p} 1(\mathrm{X})$ is executed and calls $\mathrm{q}(\mathrm{X})$, succeeding with $\mathrm{X}=\mathrm{a}$. Then, since $\mathrm{X}$ is bound, the interpreter backtracks and succeeds with $X \backslash=a$ (second clause of $q / 1)$. Now, since $\mathrm{X}$ is constrained to be different from $\mathrm{a}$, the interpreter re-executes not $\mathrm{p} 1(\mathrm{X})$ with $\mathrm{X}=\mathrm{a}$ which succeeds (first clause of $q / 1$ ). Since there are no more constrained values to be checked, the evaluation of the query finishes with success. Note that leaving $X$ unbound after the success of forall $(X, p(X))$ is consistent with the interpretation that the answer set $\{p(X)\}$ corresponds to $\forall x \cdot p(x)$.

\subsection{Non-Monotonic Checking Rules}

Non-monotonic rules are used by s(ASP) to ensure that partial stable models are consistent with the global constraints of the program. Given a consistency rule of the form $\forall \vec{x}\left(p_{i}(\vec{x}) \leftarrow \exists \vec{y} B_{i} \wedge\right.$ $\left.\neg p_{i}(\vec{x})\right)$, and in order to avoid contradictory rules of the form $p_{i}(\vec{a}) \leftarrow \neg p_{i}(\vec{a})$, all stable models must satisfy that at least one literal in $B_{i}$ is false (i.e., $\neg B_{i}$ ) or, for the values $\vec{a}$ where $B_{i}$ is true, $p_{i}(\vec{a})$ can be derived using another rule. To ensure that the partial stable model is consistent, the s(ASP) compiler generates, for each consistency rule, a rule of the form:

$$
\forall \vec{x}\left(\operatorname{chk}_{i}(\vec{x}) \longleftrightarrow \forall \vec{y}_{i}\left(\neg B_{i} \vee p(\vec{x})\right)\right)
$$

To ensure that each sub-check $\left(c h k_{i}\right)$ is satisfied, the compiler introduces into the program the rule $n m r_{-}$check $\leftarrow c h k_{1} \wedge \ldots \wedge c h k_{k}$, which is transparently called after the program query. 
Table 1. Speed comparison: $s(C A S P)$ vs. $s(A S P)$ (time in $m s)$.

\begin{tabular}{lrr}
\hline \hline & s(CASP) & s(ASP) \\
\hline hanoi $(8, T)$ & $\mathbf{1 , 5 2 8}$ & 13,297 \\
queens(4,Q) & $\mathbf{1 , 9 3 0}$ & 20,141 \\
One hamicycle & $\mathbf{4 9 3}$ & 3,499 \\
Two hamicycle & $\mathbf{3 , 6 0 5}$ & 18,026 \\
\hline \hline
\end{tabular}

\section{Example 4}

Given the program below:

$1:-\operatorname{not} s(1, X)$.

the resulting $N M R$ check rules are:

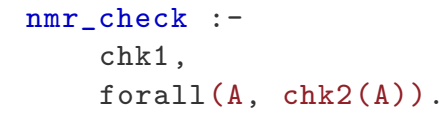

$2 \quad p(X):-q(X), \operatorname{not} p(X)$.

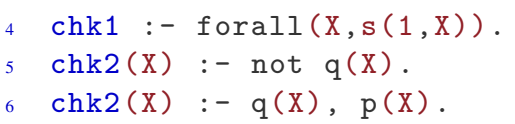

Infinite Loops Finally, in order to break infinite loops, s(ASP) uses three techniques to deal with odd loops over negation, even loops over negation, and positive loops (Marple et al. 2017b, Gupta et al. 2007). Since they are not essential for this paper, a summary is included in Appendix B of the supplementary material accompanying the paper at the TPLP archive, for the reader's convenience.

\section{3 s(CASP): Design and Implementation}

S(CASP) (available together with the benchmarks used in this paper at https://gitlab.software.imdea.org/joaquin.arias/sCASP) extends s(ASP) by computing partial stable models of programs with constraints. This extension makes the following contributions:

- The interpreter is reimplemented in Ciao Prolog (Hermenegildo et al. 2012). The driving design decision of this reimplementation is to let Prolog take care of all operations that it can handle natively, instead of interpreting them. Therefore, a large part of the environment for the s(CASP) program is carried implicitly in the Prolog environment. Since s(CASP) and Prolog shared many characteristics (e.g., the behavior of variables), this results in flexibility of implementation (see the interpreter code sketched in Figure 1 and in full in Appendix A of the supplementary material accompanying the paper at the TPLP archive) and gives a large performance improvement (Table 1). Note that all the experiments in this paper were performed on a MacOS 10.13 machine with an Intel Core i5 at $2 \mathrm{GHz}$.

- A new solver for disequality constraints.

- The definition and implementation of a generic interface to plug-in different constraint solvers. This required, in addition to changes to the interpreter, changes to the compiler which generates the dual program. This interface has been used, in this paper, to connect both the disequality constraint solver and the CLP $(\mathbb{Q})$ solver.

- The design and implementation of $C$-forall (Algorithm 2), a generic algorithm which extends the original forall algorithm (Algorithm 1) with the ability to evaluate goals with variables constrained under arbitrary constraint domains. In addition to being necessary to deal with constraints, this extension generalizes and clarifies the design of the original one. 


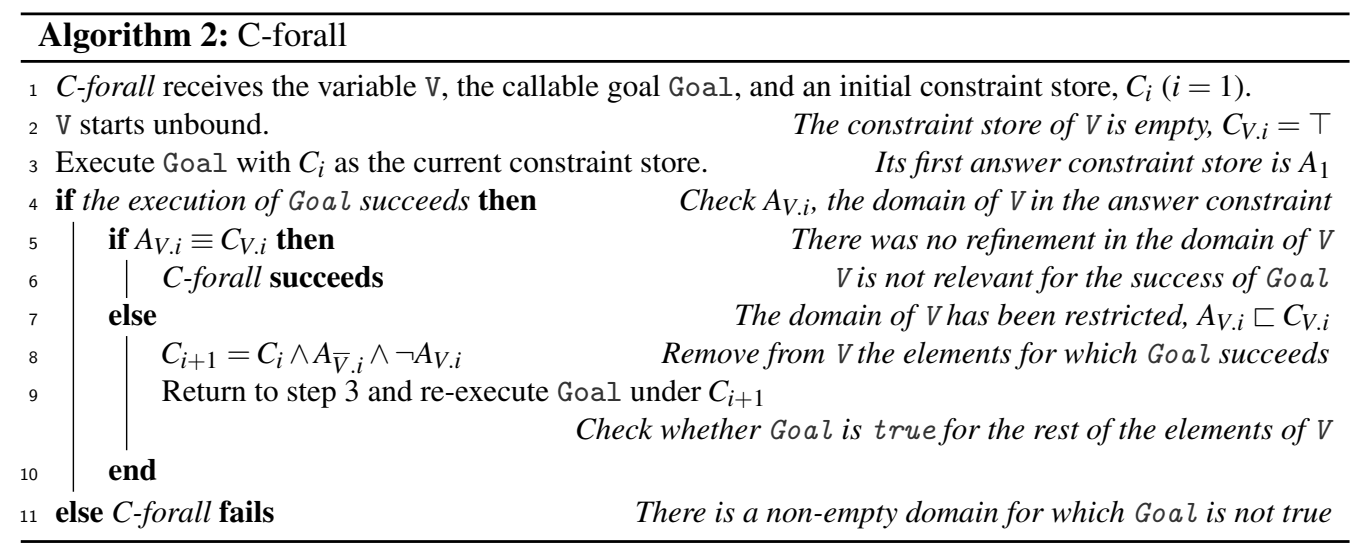

in Section 2.2; however, as mentioned before, since the solver is independent of the interpreter, its improvements are orthogonal to the core implementation of s(CASP).

The interpreter checks the call path before the evaluation of user-defined predicates to prevent inconsistencies and infinite loops (Marple et al. 2017b) (see Appendix B of the supplementary material accompanying the paper at the TPLP archive). The call path is a list constructed with the calls, and the bindings of the variables in these calls are automatically updated by Prolog.

- When a positive loops occurs, the interpreter fails only if the looping goal and its ancestor are equal (i.e., $\mathrm{p}(\mathrm{X}):-\ldots, \mathrm{p}(\mathrm{X})$ ). Termination properties are enhanced if a tabling system featuring variant calls or entailment (Arias and Carro 2016) is used as implementation target, so that all programs with a finite grounding or with the constraint-compact property terminate.

- However, when the current call is equal to an already-proven ancestor, the evaluation succeeds to avoid its re-computation and to reduce the size of the justification tree.

\subsection{Integration of Constraint Solvers in s(CASP)}

Holzbaur's CLP(Q) (Holzbaur 1995) solver was integrated in the current implementation of s(CASP). Since the interpreter already deals with the CLP $(\neq)$ constraint solver, only two details have to be taken in consideration:

- The compiler is extended to support $\operatorname{CLP}(\mathbb{Q})$ relations $\{<,>,=, \geq, \leq, \neq\}$ during the construction of the dual program and the NMR rules.

- Since it is not possible to decide at compile time whether equality will be called with $\operatorname{CLP}(\mathbb{Q})$ or Herbrand variables, its dual $\backslash=$ is extended to decide at run-time whether to call the CLP $(\mathbb{Q})$ solver or the disequality solver.

Finally, to make integrating further constraint solvers easier, the operations that the s(CASP) interpreter requires from the $\operatorname{CLP}(\mathbb{Q})$ solver are encapsulated in a single module that provides the interface between the interpreter and the constraint solver. Additional constraint solvers only need to provide the same interface. 


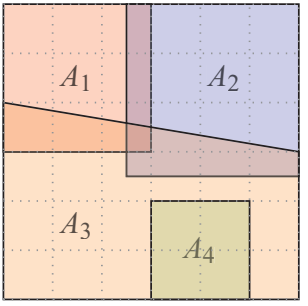

(a)

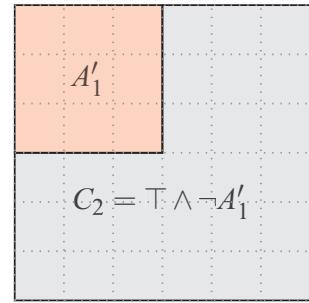

(b)

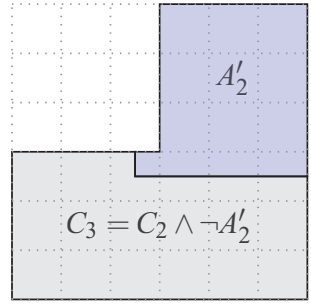

(c)

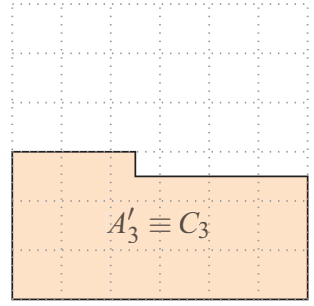

(d)

Fig. 2. A $C$-forall evaluation that succeeds.

\subsection{Extending foral l for Constraints}

Extending s(ASP) to programs with constraints requires a generalization of forall (Algorithm 1) which we will call $C$-forall (Algorithm 2). A successful evaluation of Goal in s(CASP) returns, on backtracking, a (potentially infinite) sequence of models and answer constraint stores $A_{1}, A_{2}, \ldots$ Each $A_{i}$ relates variables and constants by means of constraints and bindings (i.e., syntactical equality constraints). The execution of forall ( $\mathrm{V}, \mathrm{Goal}$ ) is expected to determine if Goal is true for all possible values of $\mathrm{V}$ in its constraint domain.

In what follows we will use $\overline{\mathrm{V}}$ to denote the set variables in Goal that are not $\mathrm{V}$ : vars(Goal) = $\{\mathrm{V}\} \cup \overline{\mathrm{V}} \wedge \mathrm{V} \notin \overline{\mathrm{V}}$. The core idea is to iteratively narrow the store $C$ under which Goal is executed by selecting one answer $A$ and re-executing Goal under the constraint store $C \wedge A_{\overline{\mathrm{V}}} \wedge \neg A_{\mathrm{V}}$, where $A_{\mathrm{V}}$ is the projection of $A$ on $\mathrm{V}$ and $A_{\overline{\mathrm{V}}}$ is the projection of $A$ on $\overline{\mathrm{V}}$. The iterative execution finishes with a positive or negative outcome.

\section{Example 5 (C-forall terminates with success)}

Figure 2 shows an example where the answers $A_{1}, \ldots, A_{4}$ to Goal cover the whole domain, represented by the square. Therefore, $C$-forall should succeed. The answer constraints that the program can generate are depicted on picture (a). For simplicity in the pictures, we will assume that the answers $A_{i}$ only restrict the domain of $\mathrm{V}$, so it will not be necessary to deal with $\mathrm{V}$ and $\overline{\mathrm{V}}$ separately since $A_{\overline{\mathrm{V}}}$ will always be empty, and therefore $A_{\mathrm{V} . i}=A_{i}$. Picture (b) shows the result of the first iteration of $C$-forall starting with $C_{1}=\top$ : answer $A_{1}$ is more restrictive than $C_{1}$ and therefore $C_{2}=C_{1} \wedge \neg A_{1}$ (in grey) is constructed. Picture (c) shows the result of the second iteration: the domain is further reduced. Finally, in picture (d) the algorithm finishes successfully because $A_{3} \equiv C_{3}$, i.e., $A_{3}$ covers the remaining domain. Note that we did not need to generate $A_{4}$.

Termination for an infinite number of answer sets The previous example points to a nice property: even if there were an infinite number of answer sets to Goal, as long as a finite subset of them covers the domain of $\mathrm{V}$ and this subset can be finitely enumerated by the program, the algorithm will finish. This is always true for constraint-compact domains, such as disequality over a finite set of constants or the gap-order constraints (Revesz 1993). Note that this happens as well in the next example, where $C$-forall fails.

\section{Example 6 (C-forall terminates with failure)}

Figure 3 shows an example where the answer constraints do not cover the domain and therefore $C$-forall ought to fail. Again, we assume that the answers $A_{i}$ only restrict the domain of V. Picture (a) depicts the answer constraints that Goal can generate. Note the gap in the domain not covered by the answers. Pictures (b) to (d) proceed as in the previous example. Picture (d) shows the final 


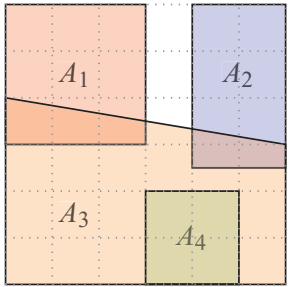

(a)

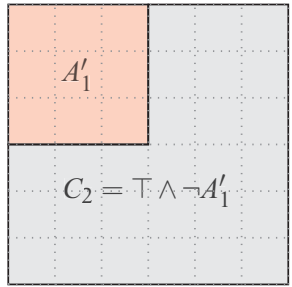

(b)

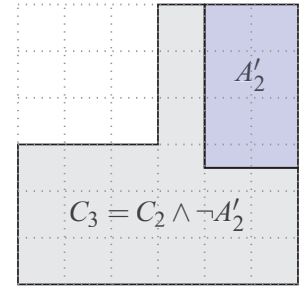

(c)

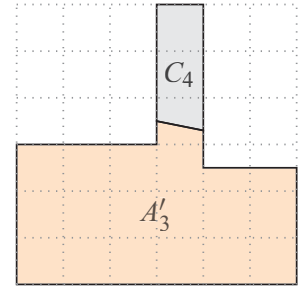

(d)

Fig. 3. A $C$-forall evaluation that fails.

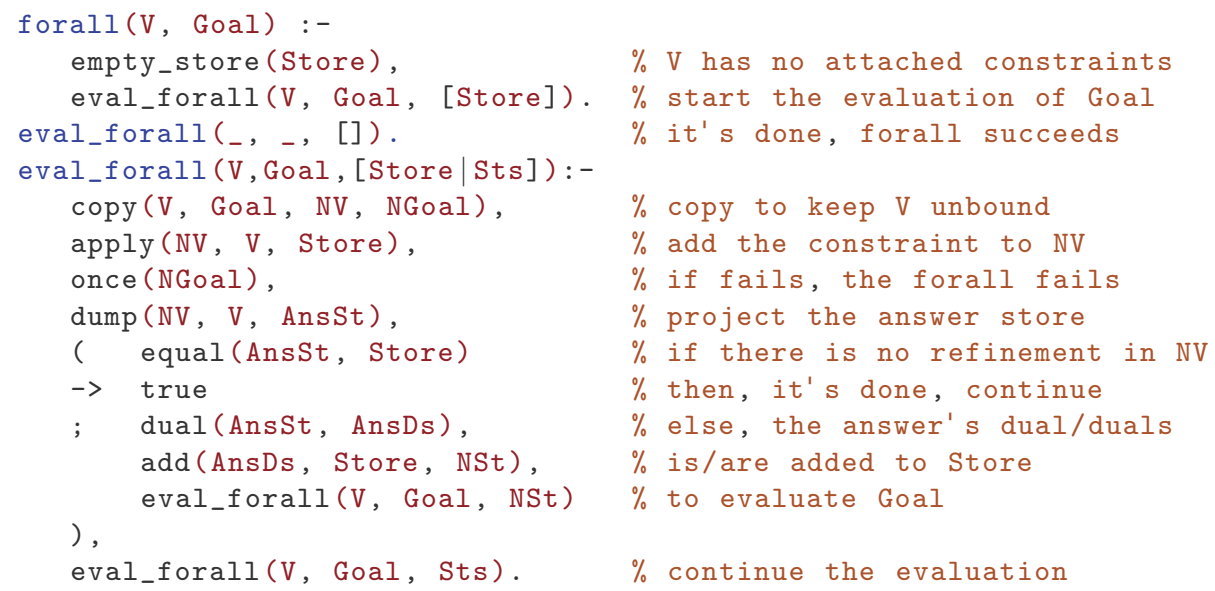

Fig. 4. Code of the predicate forall/2 implemented in s(CASP).

step of the algorithm: the execution of Goal under the store $C_{4}=C_{3} \wedge \neg A_{3}^{\prime}$ fails because the solution $A_{4}$ of Goal does not have any element in common with $C_{4}$, and then $C$-forall also fails.

Figure 4 shows a sketch of the code that implements $C$-forall in the s(CASP) interpreter, written in Prolog/CLP. In this setting, Goal carries the constraint stores $C_{i}$ and the answer stores $A_{i}$ implicitly in its execution environment. We know that the interpreter will call forall (V, Goal) with a fresh, unconstrained $\mathrm{V}$, because the executed code is generated by the s(CASP) compiler. Therefore, the projection of $C_{1}$ onto $\mathrm{V}$ is an empty constraint store, which we introduce explicitly to start the computation.

The call copy (V,Goal, NV, NGoal) copies Goal in NGoal sharing only $\overline{\mathrm{V}}$, while $\mathrm{V}$ is substituted in NGoal by a fresh variable, NV. In the main body of eval_forall/3, Store always refers to V, while NGoal does not contain V, but NV. The call apply (NV, V, Store) takes the object Store and makes it part of the global store but substituting V for NV so that the execution of NGoal can further constrain NV while V remains untouched. Note, however, that in the first iteration, NV will always remain unconstrained, since the constraint store that apply (NV,V, Store) applies to it is empty $\left(C_{\mathrm{V} .1}=\top\right)$. However, in the following iterations, Store will contain the successive constraint stores $C_{\mathrm{V} . i+1}$.

When once (NGoal) succeeds, the constraint store $C_{i} \wedge A_{\overline{\mathrm{V}} . i}$ is implicit in the binding of $\overline{\mathrm{V}}$. Therefore, the execution of eval_forall(V,Goal, Store) carries this constraint store implicitly because Goal and NGoal share $\bar{V}$. Finally, the predicate dump (NV, V, AnsSt) projects the constraint store after the execution of NGoal on NV, rewrites this projection to substitute 
$\mathrm{NV}$ for $\mathrm{V}$, and leaves the final result in AnsSt, generating $A_{\mathrm{V} . i}$. Note that, in some sense, it is transferring constraints in the opposite direction to what dump/3 did before. If the call equal (AnsSt,Store) succeeds, it means that $A_{\mathrm{V} . i} \equiv C_{\mathrm{V} . i}$ and therefore the forall succeeds (for the branch that was being explored, see below).

Otherwise, we have to negate the projection of the answer onto V, i.e., construct $\neg A_{\mathrm{V} . i}$. The negation of a conjunction generates a disjunction of constraints and most constraint solvers cannot handle disjunctions natively. Therefore, the predicate dual (AnsSt, AnsDs) returns in AnsDs a list with the components $\neg A_{\mathrm{V} . i . j}$ of this disjunction, $j=1,2, \ldots$, length(AnsDs). Then, add (AnsDs, Store, NSt) returns in NSt a list of stores, each of which is the conjunction of Store with one of the components of the disjunction in AnsDs, i.e., a list of $C_{\mathrm{V} . i} \wedge \neg A_{\mathrm{V} . i . j}$, for a fixed $i$. There may be cases where this conjunction is inconsistent; add $/ 3$ captures them and returns only the components which are consistent. Note that if a conjunction $C_{\mathrm{V} . i} \wedge \neg A_{\mathrm{V} . i . j}$ is inconsistent, it means that $\neg A_{\mathrm{V} . i . j}$ has already been (successfully) checked.

Each of the resulting constraint stores will be re-evaluated by eval_forall/3, where apply/3 will apply them to a new variable NV, in order to complete the implicit construction of $C_{i+1}$ before the execution of once (NGoal). forall/2 finishes with success when there are no pending constraint stores to be processed (line 4).

\section{Example 7 ( $C$-foral - execution negating a constraint conjunction)}

Given the program below, consider the evaluation of forall $(A, p(A))$ :

$$
\begin{array}{llll}
1 & \mathrm{p}(\mathrm{X}):-\mathrm{X} \#>=0, \mathrm{X} \#=<5 . & 3 \mathrm{p}(\mathrm{X}):-\mathrm{X} \#<3 . \\
2 & \mathrm{p}(\mathrm{X}):-\mathrm{X} \#>1 . & 4 \mathrm{p}(\mathrm{X}):-\mathrm{X} \#<1 .
\end{array}
$$

In the first iteration $C_{1}=\top$. The first answer is $A_{1}=\{X \geq 0 \wedge X \leq 5\}$, which is more restrictive than $C_{1}$, so we compute $\neg A_{1}=\{X<0 \vee X>5\}$. First, p/1 is evaluated with $C_{2 . a}=\{\top \wedge X<0\}$ obtaining $A_{2 . a}=\{X<0\}$ using the third clause. Since $A_{2 . a} \equiv C_{2 . a}$, we are done with $C_{2 . a}$. But we also have to evaluate $\mathrm{p} / 1$ with $C_{2 . b}=\{\top \wedge X>5\}$. Using the second clause, $A_{2 . b}=\{X>5\}$ is obtained and since $A_{2 . b} \equiv C_{2 . b}$, the evaluation succeeds.

\section{Examples and Evaluation}

The expressiveness of s(CASP) allows the programmer to write programs / queries that cannot be written in [C]ASP without resorting to a complex, unnatural encoding. Additionally, the answers given by $\mathrm{s}(\mathrm{CASP})$ are also more expressive than those given by ASP. This arises from several points:

- $\mathrm{s}(\mathrm{CASP})$ inherits from $\mathrm{s}(\mathrm{ASP})$ the use of unbound variables during the execution and in the answers. This makes it possible to express constraints more compactly and naturally (e.g., ranges of distances can be written using constraints)

- $\mathrm{s}(\mathrm{CASP})$ can use structures / functors directly, thereby avoiding the need to encode them unnaturally (e.g., giving numbers to Hanoi movements to represent what in a list is implicit in the sequence of its elements).

- The constraints and the goal-directed evaluation strategy of s(CASP) makes it possible to use direct algorithms and to reduce the search space (e.g., by putting bounds on a path's length). 
apply partial evaluation and better compilation techniques to remove (part of) the overhead brought about by the interpreting approach.

\section{Supplementary materials}

For supplementary material for this article, please visit https://doi.org/10.1017/ S1471068418000285

\section{References}

Alferes, J. J., Pereira, L. M., AND Swift, T. 2004. Abduction in Well-Founded Semantics and Generalized Stable Models via Tabled Dual Programs. Theory and Practice of Logic Programming 4, 4, 383-428.

Alviano, M., Faber, W., Greco, G., And Leone, N. 2012. Magic Sets for Disjunctive Datalog Programs. Artificial Intelligence 187, 156-192.

ARIAS, J. 2016. Tabled CLP for Reasoning over Stream Data. In Technical Communications of the 32nd Int'l Conference on Logic Programming (ICLP'16). Vol. 52. OASIcs, 1-8. Doctoral Consortium.

ARIAS, J. AND CARro, M. 2016. Description and Evaluation of a Generic Design to Integrate CLP and Tabled Execution. In 18th Int'l. ACM SIGPLAN Symposium on Principles and Practice of Declarative Programming (PPDP'16). ACM Press, 10-23.

BAlduccini, M. AND Lierler, Y. 2017. Constraint Answer Set Solver EZCSP and why Integration Schemas Matter. Theory and Practice of Logic Programming 17, 4, 462-515.

Banbara, M., Kaufmann, B., Ostrowski, M., And Schaub, T. 2017. Clingcon: The Next Generation. Theory and Practice of Logic Programming 17, 4, 408-461.

Baselice, S. And Bonatti, P. A. 2010. A Decidable Subclass of Finitary Programs. Theory and Practice of Logic Programming 10, 4-6, 481-496.

Baselice, S., Bonatti, P. A., And Criscullo, G. 2009. On Finitely Recursive Programs. Theory and Practice of Logic Programming 9, 2, 213-238.

Brewka, G., Eiter, T., And Truszczyński, M. 2011. Answer Set Programming at a Glance. Communications of the ACM 54, 12, 92-103.

Calimeri, F., Cozza, S., And IAnni, G. 2007. External Sources of Knowledge and Value Invention in Logic Programming. Annals of Mathematics and Artificial Intelligence 50, 3-4, 333-361.

Clark, K. L. 1978. Negation as Failure. In Logic and Data Bases, H. Gallaire and J. Minker, Eds. Plenum.

Dal Palù, A., Dovier, A., Pontelli, E., And Rossi, G. 2009. GASP: Answer Set Programming with Lazy Grounding. Fundamenta Informaticae 96, 3, 297-322.

Dovier, A., Formisano, A., And Pontelli, E. 2005. A Comparison of CLP(FD) and ASP Solutions to NP-Complete Problems. In International Conference on Logic Programming. Springer, 67-82.

GabBrielli, M. And Levi, G. 1991. Modeling Answer Constraints in Constraint Logic Programs. In Proc. Eighth Int'l Conf. on Logic Programming.

Gebser, M., Kaminski, R., Kaufmann, B., Ostrowski, M., Schaub, T., And Thiele, S. 2008. A User's Guide to gringo, clasp, clingo, and iclingo.

Gelfond, M. And Lifschitz, V. 1988. The Stable Model Semantics for Logic Programming. In International Conference on Logic Programming 1988. 1070-1080.

Gelfond, M. AND Lifschitz, V. 1991. Classical Negation in Logic Programs and Disjunctive Databases. New Generation Computing 9, 3/4, 365-386.

Gupta, G., Bansal, A., Min, R., Simon, L., And Mallya, A. 2007. Coinductive Logic Programming and its Applications. Logic Programming, 27-44.

Hermenegildo, M. V., Bueno, F., Carro, M., López, P., Mera, E., Morales, J., And Puebla, G. 2012. An Overview of Ciao and its Design Philosophy. Theory and Practice of Logic Programming 12, 1-2 (January), 219-252. http://arxiv.org/abs/1102.5497. 
Hölldobler, S. ANd Schweizer, L. 2014. Answer Set Programming and clasp, a Tutorial. In Young Scientists' International Workshop on Trends in Information Processing (YSIP). 77.

Holzbaur, C. 1995. OFAI CLP(Q,R) Manual, Edition 1.3.3. Tech. Rep. TR-95-09, Austrian Research Institute for Artificial Intelligence, Vienna.

JAfFAR, J. AND MAher, M. 1994. Constraint Logic Programming: A Survey. Journal of Logic Programming 19/20, 503-581.

Janhunen, T., Kaminski, R., Ostrowski, M., Schellhorn, S., Wanko, P., and Schaub, T. 2017. Clingo goes Linear Constraints over Reals and Integers. TPLP 17, 5-6, 872-888.

Ji, J., Wan, H., WANG, K., WANG, Z., Zhang, C., AND XU, J. 2016. Eliminating Disjunctions in Answer Set Programming by Restricted Unfolding. In IJCAI. 1130-1137.

Marple, K., Salazar, E., Chen, Z., And Gupta, G. 2017a. The s(ASP) Predicate Answer Set Programming System. The Association for Logic Programming Newsletter.

Marple, K., Salazar, E., And Gupta, G. 2017b. Computing Stable Models of Normal Logic Programs Without Grounding. CoRR abs/1709.00501.

Revesz, P. Z. 1993. A Closed-Form Evaluation for Datalog Queries with Integer (Gap)-Order Constraints. Theoretical Computer Science 116, 1, 117-149. 